\title{
Red deer synchronise their activity with close neighbours
}

Models of collective animal behaviour frequently make assumptions about the effects of neighbours on the behaviour of focal individuals, but these assumptions are rarely tested.

One such set of assumptions is that the switch between active and inactive behaviour seen in herding animals is influenced by the activity of close neighbours, where neighbouring animals show a higher degree of behavioural synchrony than would be expected by chance. We tested this assumption by observing the simultaneous behaviour of paired individuals within a herd of red deer Cervus elaphus. Focal individuals were more synchronised with their two closest neighbours than with the third closest or randomly selected individuals from the herd. Our results suggest that the behaviour of individual deer is influenced by immediate neighbours. Even if we assume that there are no social relationships between individuals, this suggests that the assumptions made in models about the influence of neighbours may be appropriate. 


\section{Red deer synchronise their activity with close neighbours}

2 Sean A. Rands*, Hayley Muir†, and Naomi L. Terry†

$3 \uparrow \mathrm{HM}$ and NLT contributed equally to this work

4 *author for correspondence

5 School of Biological Sciences, University of Bristol, Bristol, UK 
7 Many animals form groups at some point in their life cycle. In most cases, these groups occur

8 because there is some benefit from being in the group to each of its members (Krause \& Ruxton

9 2002), suggesting that the behaviour of each individual must in part be both influenced by and

10 directed towards behaving as part of the group. Models of collective behaviour (Camazine et al.

11 2001; Sumpter 2010) frequently consider the behaviours of groups that emerge from the

12 combined actions of the individuals within the group. These models are good at creating

13 simulations of the movements and decision-making processes of groups that appear to behave in

14 very similar ways to what is seen in nature, but very different models can produce similar

15 phenomena. In order to identify which modelled processes are appropriate, it is essential to

16 challenge these models with empirical data. However, the noisiness of biological systems (either

17 from observational error or biological variation between individuals) increases the difficulty of

18 testing whether the interaction rules used in these models are appropriate (Mann 2011).

19 Many of the models and associated empirical studies that describe collective behaviour typically

20 consider individuals that are influenced by other group members who are in close proximity,

21 either within a physical 'metric' distance of a focal individual (Couzin et al. 2002; Herbert-Read

22 et al. 2011; Rands et al. 2004; Rands et al. 2006; Romey \& Vidal 2013), or according to a

23 topologically-defined network of interacting individuals (Bode et al. 2011a; Camperi et al. 2012;

24 Nagy et al. 2010). Other influential models of movement involve changes in behavioural states,

25 considering the departure and leadership decisions made by groups of moving animals

26 (Fernandez \& Deneubourg 2011; Pillot et al. 2011; Sueur et al. 2011), where the behavioural state

27 change experienced by individuals is the switch from being static to moving. Other studies of

28 behavioural state changes have considered how local interactions govern changes between being 
29 vigilant and non-vigilant (Beauchamp et al. 2012), or being active and inactive according to both

30 social facilitation and metabolic requirements (Ruckstuhl \& Kokko 2002).

31 Given this wide variety of models exploring collective behaviour, empirical tests exploring the

32 individual behaviours driving observed collective behaviours are patchy in their coverage. Much

33 research effort has been devoted to exploring how decision-making and leadership processes are

34 connected and distributed within groups (Conradt \& List 2009; Dyer et al. 2009; King \&

35 Cowlishaw 2009). Specific consideration of the effects of inter-neighbour interactions have

36 explored individual decisions made during group movement according to metric (Herbert-Read et

37 al. 2011; Ramseyer et al. 2009) or topological distance to neighbours (Ballerini et al. 2008; Nagy

38 et al. 2010), and there have been a number of studies exploring leaving decisions (Sueur et al.

39 2011). Fewer studies have considered changes in behavioural state within a group. Several have

40 considered how neighbours influence the vigilance patterns of groups (Beauchamp 2009). Most

41 tests of the models exploring changes in activity in response to metabolic requirements and the

42 behaviour of neighbours (Ruckstuhl \& Kokko 2002) have focused on how difference in energetic

43 requirements can lead to sexual segregation (Aivaz \& Ruckstuhl 2011; Michelena et al. 2008;

44 Yearsley \& Pérez-Barberia 2005), non-synchronous behaviour (Šárová et al. 2007), group

45 cohesion (Conradt 1998), and group-size effects on activity (Gautrais et al. 2007). However,

46 although these models assume that behavioural state is influenced by the actions of close

47 neighbours, little has been done to test this empirically. Evidence is suggested by a study of cattle

48 Bos taurus synchronising their lying behaviour, where their posture is more likely to be similar to

49 neighbouring individuals compared to the rest of the herd (Stoye et al. 2012). However, there is

50 scope for much more exploration of the assumptions behind models considering how the

51 proximity of individuals to others can influence switches in their behavioural state. In this study,

52 we asked whether the behaviours of individual red deer Cervus elaphus living in a managed herd 
53 are influenced by their neighbours. Individual deer spend large parts of their lives near or within

54 large herds (Clutton-Brock \& Albon 1989), and therefore are ideal for addressing how changes in

55 individual activity tie in with group-level behaviour. We hypothesised that deer that were

56 topologically closer within the herd were more likely to be synchronised than would be expected

57 when comparing two individuals randomly selected from different locations within the herd.

58 MATERIALS AND METHODS

59 The work described is purely observational, conforming with UK law and ASAB/ABS guidelines

60 on animal experimentation. Ethical approval was given by the University of Bristol Ethical

61 Review Group (University Investigation Number UB/12/035).

62 The herd studied was housed in an enclosed 40.5 hectare deer park in the Ashton Court Estate,

63 Bristol, England, composed of open grassland, with scattered patches of woodland. The herd is a

64 population of c. 99 individuals of mixed age and sex, and its management and husbandry is

65 conducted by Bristol City Council (the exact herd size was not known at the time of observation).

66 Except for rutting periods, the enclosure is accessible to the general public, and the deer are

67 habituated to the presence of humans and dogs. Permissions were not required for these

68 observational studies, which occurred during the hours the public had access to the park. All

69 observations were conducted within $10-100 \mathrm{~m}$ of the focal individuals, using binoculars where

70 appropriate: for habituation, observers were in position for recording at least five minutes before

71 observations started.

73 The study coincided with the rutting season of the deer, with stags often solitary and with greatly 
74 reduced feeding, and therefore likely to display very different behaviours to the rest of the herd

75 (Clutton-Brock \& Albon 1989; Pépin et al. 2009). Males with antlers (approximately eleven

76 individuals) were therefore excluded from the observations. The study focussed on females and

77 young males that had not yet segregated from their maternal group, which were likely to display

78 behaviour similar to the females (Clutton-Brock \& Albon 1989).

79 Prior to the study described, an ethogram was constructed for individual behaviour within the

80 herd, differentiating between grazing, standing, walking, running, interacting, laying with head

81 down, laying with head alert, and laying whilst ruminating. Within the analysis, these were

82 reclassified as a combined dichotomous behaviours. Individuals were classified as 'active' if they

83 were grazing, standing, walking, running, and interacting, and 'resting' if they were conducting

84 one of the other behaviours.

85 For a single observation period, a focal individual was randomly selected from the herd. A

86 random number between 1 and 99 was generated, and, considering the visible deer in the

87 observer's field of vision, the focal deer was selected by counting linearly from leftmost or

88 rightmost visible deer (where the direction of counting was selected by a coin toss, and where a

89 count was discarded if the random number selected was larger than the number of deer visible:

90 this randomisation technique may have introduced some unavoidable bias towards individuals on

91 the side of the herd closest to the observer, but, ignoring outlying stags, most of the herd was

92 visible and countable during the sampling period and this bias should therefore have been

93 minimal). Selected focal individuals were watched for twenty minutes. If the herd was disturbed

94 by a human presence in the middle of the observation period, the observation was aborted and the

95 data discarded. In total, eighteen complete observations of twenty minutes were conducted, over

96 four days in October 2012; an additional two planned observations were started but aborted early 
97 due to disturbance, and have not been included in the analysis. All observations were conducted

98 within 1200 and $1630 \mathrm{~h}$, outside of the dawn and dusk peaks of activity frequently shown by red 99 deer (Clutton-Brock et al. 1982).

100 Over an observation period, the behaviour of the focal individual was recorded every minute.

101 Simultaneously, the behaviour of the first, second and third closest individual in the herd to the

102 focal were also recorded (where the identities of these individuals could change between the

103 recording events as the deer moved within the herd). At the same time, the behaviour of a

104 different randomly selected control individual within the herd was also recorded (selected using

105 the randomisation technique described above from what remained of the herd after the focal and

106 three nearest neighbours had been excluded, and ignoring rutting stags as stated above), where

107 the identity of the control individual was independently chosen at each recording event.

108 Synchronisation between individuals was calculated as the proportion of the observations where

109 the focal and test individual were both active or both inactive. Data did not follow the normality

110 assumptions necessary for a repeated-measures analysis of variance, and were therefore

111 compared with Freidman tests (Friedman 1937). Because there was some change that focal

112 individuals were re-selected by chance, there could be some degree of pseudoreplication in the

113 dataset. To explore this, we generated a full set of Friedman tests where all possible combinations

114 of up to five of the focal individuals were excluded from the analysis. Post-hoc tests were

115 conducted for the comparison of synchronisation at different proximities, using two-tailed

116 Wilcoxon signed-ranks tests assuming a normal approximation with continuity corrections, with

117 the significance value adjusted to $p=0.009$ using a Bonferroni correction. All analyses were

118 conducted with $R 3.0$ ( R Development Core Team 2013). Raw data are presented in Supplemental

119 Information 1. 


\section{RESULTS AND DISCUSSION}

121 Deer are less likely to be synchronised as they become socially further away from a focal

122 individual $\left(\chi_{3}^{2}=21.36, p<0.001\right.$; Fig. 1$)$. Because deer could not be individually identified, there

123 is some chance that some pseudoreplication has occurred, with focal deer being resampled by

124 chance. However, randomly removing data (all possible combinations of up to five focal

125 individuals were removed) had no effect upon these results (the range of $p$ values obtained fell in

126 the range $0.000005-0.018)$. Post hoc tests demonstrated that focal individuals were more

127 synchronised with first and second closest neighbours than with control deer (Fig. 1), but the

128 increased synchronisation with the third-closest neighbour compared to the control $(p=0.011)$

129 was not significant after applying Bonferroni corrections.

130 We used a dichotomous classification for behaviour, following the differentiation between

131 'active' and 'passive' behaviours used by Ruckstuhl \& Kokko (2002). Individual deer were active

132 for $73.11 \%$ of their time during the period observed (calculated by combining the individual

133 datasets collected for focal, neighbour and control individuals: most of this active behaviour was

134 grazing behaviour, as can be seen in Supplemental Information 2). If we assumed that all deer

135 were acted independently of each other, then we can estimate that if we were to pick two

136 individuals at random, they would be conducting the same action $60.68 \%$ of the time. This

137 corresponds with the dotted line shown in Fig. 1, which falls near the middle of the control

138 results. The three close neighbours were much more likely to be synchronised than this random

139 estimate, suggesting that their individual behaviours are at least partially influenced by each 
140 other. The dichotomous scheme that we use may be falsely classifying some behaviours as

141 similar (e.g. one member of an 'active' pair might be grazing whilst its partner is running).

142 However, our dichotomous classification follows the differentiation between 'active' and

143 'passive' behaviours used by Ruckstuhl \& Kokko (2002), which they demonstrate are sufficient

144 to drive movement and segregation processes in ungulate-like animals. We would suggest that

145 individuals conducting resting behaviours may have to invest more energy and expose

146 themselves to a potentially greater risk of predation if they have to suddenly switch to one of the

147 'active' behaviours than if they were switching between two different 'active' behaviours or two

148 different 'resting' behaviours. Therefore, considering just two behavioural states may be

149 sufficient to try and pick apart broad patterns of synchronisation. Considering two easily-

150 distinguished states also means that we are less likely to incorrectly classify finer-scale

151 behaviours in the field that could look similar (such as the different 'resting' behaviours we

152 initially recorded), although we do note that similar results are gained if we ignore this

153 dichotomous classification and consider the exact synchronisation of the eight behaviour types

154 recorded (Supplemental Information 3).

155 In considering the three nearest neighbours to a focal individual at a given moment in time, it was

156 necessary to ignore a few factors which may have an effect on each individual's behaviour.

157 Firstly, the identity of each neighbour is likely to have changed over the course of consecutive

158 observations of a focal deer. However, if we are interested in demonstrating that proximity is a

159 factor driving behavioural synchronisation, this is not an issue as it is how the actions of the focal

160 individual correlate with its unidentified neighbours that is important. Secondly, the observations

161 do not account for inter-neighbour distance, where individuals in physically close proximity may

162 be more likely to be synchronised. However, we are considering a topological relationship here

163 (as is considered by Ballerini et al. 2008; Nagy et al. 2010) rather than a metric distance: it would 
164 be illuminating to observe whether increased physical proximity increases synchronisation, but

165 the logistics of field observation made this too difficult to observe accurately. Thirdly, because 166 this is an observational study, we are unable to separate whether synchronisation of activity is

167 occurring in response to neighbour behaviour from whether some local effect is driving the

168 behaviour instead: for example, deer that are close together may be more likely to be grazing

169 because the quality of the local patch of grass available to them is better than that experienced by

170 more distant individuals. Similarly, because we are looking at correlations, we are unable to

171 separate mechanisms that may be causing local synchronisation from the observed behaviour:

172 synchronisation could be occurring because key individuals are driving the local behaviours

173 within the herd (King \& Cowlishaw 2009; Rands 2011). To move from observing correlations to

174 picking apart how synchronisation works, we would need to conduct experimental manipulations

175 of the herd, such as by changing local forage quality or by removing possible key individuals

176 from the herd.

177 The synchronisation behaviour we describe does not account for social relationships between the

178 individuals. Local social networks are likely to strongly influence substructures within groups

179 (Bode et al. 2011b; Sueur et al. 2011), and being able to identify individuals and assay their

180 interaction behaviour over longer periods of time may give us a much clearer indication of the

181 behavioural dynamics of the herd. Similarly, we did not account for how differences in the

182 physiological state ( $\underline{\text { Rands et al. 2003; }} \underline{\text { Rands et al. 2008; }} \underline{\text { Rands et al. 2006) }}$ ) or social status

183 (Rands 2011) of individuals could be influencing their need to copy the behaviour of others.

184 There is still a need to properly link models and empirical work considering how social foraging

185 behaviour can influence group behaviour (Marshall et al. 2012), and in particular we urge further

186 studies of the effects of neighbour proximity in order to explore these neglected assumptions

187 implicit within many models. 
188 This study demonstrates that individual deer are more likely to synchronise their activity with

189 their closer neighbours when compared to more distant neighbours and the wider herd. This

190 provides support for the spatial assumptions used in models of activity synchronisation

191 (Ruckstuhl \& Kokko 2002). Similar patterns were seen in small herds of cattle (Stoye et al.

192 2012), but the current study demonstrates that these assumptions may also be applicable to much

193 larger herds of animals.

\section{ACKNOWLEDGEMENTS}

195 Andrew Robins, Benjamin Dalziel, and four anonymous referees who commented on earlier 196 versions of this manuscript are thanked.

199 Data presented as a comma-delimited file: full details of how the data is encoded is given at the 200 beginning of the file.

201 Supplemental Information 2: Figure showing overall proportion of observations where deer 202 were conducting each of the classified behaviours. 
focal individual was synchronised with neighbours of differing social distances, considering

205 the exact behaviour the focal is conducting.

206 Synchronisation of individual behaviours between the focal individual and its neighbours or a

207 control individual was assessed by calculating the proportion of observation periods that each

208 focal individual was conducting exactly the same behaviour as the compared individual,

209 considering each of the eight possible behavioural classes recorded as a different behaviour. Data

210 were compared using a Friedman test, as they did not fit assumptions of normality, and post hoc

211 analyses were conducted using two-tailed Wilcoxon signed-ranks tests assuming a normal

212 approximation with continuity corrections, with the significance value adjusted to $p=0.009$

213 using a Bonferroni correction. Deer at different social distances differed in their level of

214 synchronisation with the focal individual $\left(\chi^{2}=19.29, p<0.001\right)$, and pairwise comparisons

215 demonstrated that the focal individuals were more likely to be synchronised with their closest

216 neighbour than with a control individual $(p=0.001)$; all other pairwise comparisons were non-

217 significant. 
219 Aivaz AN, and Ruckstuhl KE. 2011. Costs of behavioral synchrony as a potential driver behind size-assorted grouping. Behavioral Ecology 22:1353-1363.

221 Ballerini M, Cabibbo N, Candelier R, Cavagna A, Cisbani E, Giardina I, Lecomte V, Orlandi A, sexes. Chicago: Chicago University Press. 
242 Conradt L. 1998. Could asynchrony in activity between the sexes cause intersexual social 243 segregation in ruminants? Proceedings of the Royal Society B 265:1359-1363.

244 Conradt L, and List C. 2009. Group decisions in humans and animals: a survey. Philosophical 245 Transactions of the Royal Society B 364:719-742.

246 Couzin ID, Krause J, James R, Ruxton GD, and Franks NR. 2002. Collective memory and spatial 247 sorting in animal groups. Journal of Theoretical Biology 218:1-11.

248 Dyer JRG, Johansson A, Helbing D, Couzin ID, and Krause J. 2009. Leadership, consensus 249 decision making and collective behaviour in humans. Philosophical Transactions of the Royal Society B 364:781-789.

251 Fernandez AA, and Deneubourg J-L. 2011. On following behaviour as a mechanism for 252 collective movement. Journal of Theoretical Biology 284:7-15.

253 Friedman M. 1937. The use of ranks to avoid the assumption of normality in the analysis of 254 variance. Journal of the American Statistical Association 32:675-701.

Gautrais J, Michelena P, Sibbald A, Bon R, and Deneubourg J-L. 2007. Allelomimetic synchronization in Merino sheep. Animal Behaviour 74:1443-1454.

Herbert-Read JE, Perna A, Mann RP, Schaerf TM, Sumpter DJT, and Ward AJW. 2011. Inferring 258 the rules of interaction of shoaling fish. Proceedings of the National Academy of Sciences

King AJ, and Cowlishaw G. 2009. Leaders, followers, and group decision-making. Communicative and Integrative Biology 2:147-150.

262 Krause J, and Ruxton GD. 2002. Living in groups. Oxford: Oxford University Press.

263 Mann RP. 2011. Bayesian inference for identifying interaction rules in moving animal groups.

$264 \quad$ PLoS One 6:e22827.

265 Marshall HH, Carter AJ, Rowcliffe JM, and Cowlishaw G. 2012. Linking social foraging 266 behaviour with individual time budgets and emergent group-level phenomena. Animal 
268 Michelena P, Gautrais J, Gérard J-F, Bon R, and Deneubourg J-L. 2008. Social cohesion in groups of sheep: effect of activity level, sex composition and group size. Applied Animal Behaviour Science 112:81-93.

Nagy M, Ákos Z, Biro D, and Vicsek T. 2010. Hierarchical group dynamics in pigeon flocks. Nature 464:890-893. Vienna: R Foundation for Statistical Computing.

Rands SA, Cowlishaw G, Pettifor RA, Rowcliffe JM, and Johnstone RA. 2003. The spontaneous emergence of leaders and followers in a foraging pair. Nature 423:432-434.

Rands SA, Cowlishaw G, Pettifor RA, Rowcliffe JM, and Johnstone RA. 2008. The emergence of leaders and followers in foraging pairs when the qualities of individuals differ. $B M C$ Evolutionary Biology 8:51.

289 Rands SA, Pettifor RA, Rowcliffe JM, and Cowlishaw G. 2004. State-dependent foraging rules for social animals in selfish herds. Proceedings of the Royal Society B 271:2613-2620.

291 Rands SA, Pettifor RA, Rowcliffe JM, and Cowlishaw G. 2006. Social foraging and dominance 

Sociobiology 60:572-581.

Romey WL, and Vidal JM. 2013. Sum of heterogeneous blind zones predict movements of simulated groups. Ecological Modelling 258:9-15.

296 Ruckstuhl KE, and Kokko H. 2002. Modelling sexual segregation in ungulates: effects of group size, activity budgets and synchrony. Animal Behaviour 64:909-914.

298 Šárová R, Špinka M, and Panamá JLA. 2007. Synchronization and leadership in switches between resting and activity in a beef cattle herd - a case study. Applied Animal Behaviour Science 108:327-331.

301 Stoye S, Porter MA, and Dawkins MS. 2012. Synchronized lying in cattle in relation to time of 302 day. Livestock Science 149:70-73.

303 Sueur C, King AJ, Conradt L, Kerth G, Lusseau D, Mettke-Hofmann C, Schaffner CM, Williams

304 LM, Zinner D, and Aureli F. 2011. Collective decision-making and fission-fusion 305 dynamics: a conceptual framework. Oikos 120:1608-1617.

306 Sumpter DJT. 2010. Collective animal behavior. Princeton: Princeton University Press.

307 Yearsley JM, and Pérez-Barberia FJ. 2005. Does the activity budget hypothesis explain sexual 308 segregation in ungulates? Animal Behaviour 69:257-267. 


\section{Figure 1}

Boxplot showing the proportion of time that behaviour of the focal individual was synchronised with neighbours of differing social distances.

Significant pairwise post-hoc tests are shown.

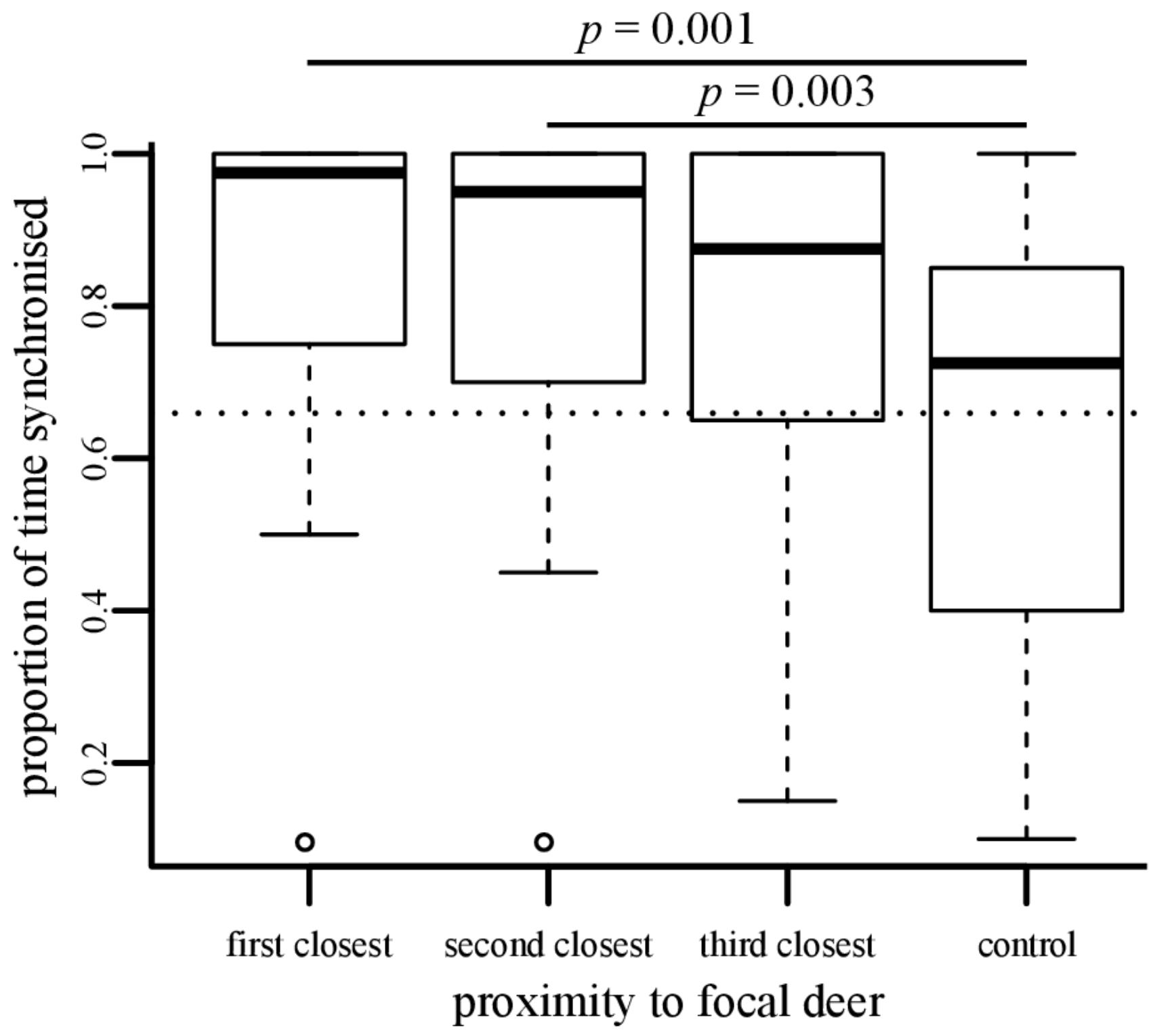

